OPEN ACCESS

Edited by:

Ovidiu Constantin Baltatu,

Anhembi Morumbi University, Brazil

Reviewed by:

Jiin-Cherng Yen,

National Yang-Ming University, Taiwan

Paul Kenneth Witting,

University of Sydney, Australia

*Correspondence:

Ching-Yi Tsai

cytsai@cgmh.org.tw

Specialty section: This article was submitted to Integrative Physiology, a section of the journal

Frontiers in Physiology

Received: 31 January 2019

Accepted: 03 June 2019

Published: 18 June 2019

Citation:

Chen S-M, Phuagkhaopong S,

Fang $C$, Wu JCC, Huang $Y-H$, Vivithanaporn P, Lin H-H and Tsai C-Y (2019) Dose-Dependent Acute Circulatory Fates Elicited by Cadmium

Are Mediated by Differential Engagements of Cardiovascular Regulatory Mechanisms in Brain. Front. Physiol. 10:772 doi: 10.3389/fphys.2019.00772

\section{Dose-Dependent Acute Circulatory Fates Elicited by Cadmium Are Mediated by Differential Engagements of Cardiovascular Regulatory Mechanisms in Brain}

\author{
Shu-Mi Chen ${ }^{1,2,3}$, Suttinee Phuagkhaopong ${ }^{4}$, Chi Fang ${ }^{3}$, Jacqueline C. C. Wu ${ }^{3}$, \\ Ya-Hui Huang ${ }^{3}$, Pornpun Vivithanaporn ${ }^{4}$, Hsun-Hsun Lin ${ }^{5}$ and Ching-Yi Tsai ${ }^{3 *}$

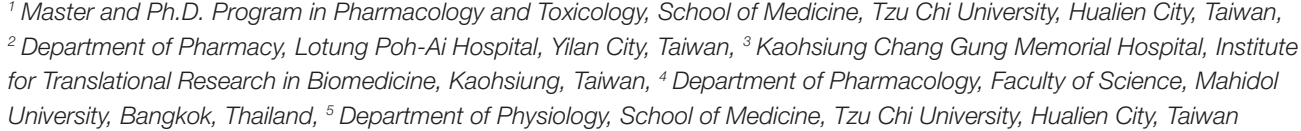

Master and Ph.D. Program in Pharmacology and Toxicology, School of Medicine, Tzu Chi University, Hualien City, Taiwan, ${ }^{2}$ Department of Pharmacy, Lotung Poh-Ai Hospital, Yilan City, Taiwan, ${ }^{3}$ Kaohsiung Chang Gung Memorial Hospital, Institute for Translational Research in Biomedicine, Kaohsiung, Taiwan, ${ }^{4}$ Department of Pharmacology, Faculty of Science, Mahidol University, Bangkok, Thailand, ${ }^{5}$ Department of Physiology, School of Medicine, Tzu Chi University, Hualien City, Taiwan

Whereas cadmium is a toxicant that has been shown to cause cardiovascular toxicity and mortality in mammals, few mechanistic studies address its acute circulatory actions. The present study assessed the hypothesis that cadmium effects dose-dependent acute circulatory fates via differential participation of the cardiovascular regulatory mechanisms in brain. In Sprague-Dawley rats maintained under propofol anesthesia, cadmium acetate $(8 \mathrm{mg} / \mathrm{kg}$, iv) induced significantly high mortality rate within $10 \mathrm{~min}$, concomitant with progressive decline toward zero level of mean arterial pressure (MAP), heart rate (HR), baroreflex-mediated sympathetic vasomotor tone, and carotid blood flow (CBF). There were concurrent tissue anoxia, cessation of microvascular perfusion, reduction of mitochondrial membrane potential and ATP production, and necrotic cell death in the rostral ventrolateral medulla (RVLM), the brain stem site that maintains blood pressure and sympathetic vasomotor tone. On the other hand, a lowerdose of cadmium (4 mg/ $/ \mathrm{kg}$, iv) resulted in only a transient decrease in MAP that was mirrored by an increase in CBF and baroreflex-mediated sympathetic vasomotor tone, minor changes in HR, along with transient hypoxia, and apoptotic cell death in RVLM. We conclude that cadmium elicits dose-dependent acute cardiovascular effects with differential underlying biochemical and neural mechanisms. At a higherdose, cadmium induces high mortality by effecting acute cardiovascular collapse via anoxia, diminished tissue perfusion, mitochondrial dysfunction and bioenergetics failure 
that echo failure of cerebral autoregulation, leading to necrosis, and loss of functionality in RVLM. On the other hand, a lower-dose of cadmium elicits low mortality, transient decrease in arterial pressure, and hypoxia and apoptosis in RVLM that reflect sustained cerebral autoregulation.

Keywords: cadmium, bioenergetic failure, anoxia, rostral ventrolateral medulla, cerebral autoregulation, mitochondrial membrane potential

\section{INTRODUCTION}

Cadmium is a heavy metal and an environmental toxicant. Acute cadmium exposure induces cardiovascular toxicity that may lead to mortality (Dote et al., 2007; Miura et al., 2012). A majority of the mechanistic reports on cardiovascular toxicity elicited by cadmium is based on either long-term exposure to this toxicant (Puri, 1999; Yoopan et al., 2008; Ozturk et al., 2009) or on exposure to cadmium in vitro (Majumder et al., 2008; Messner and Bernhard, 2010; Messner et al., 2016) or ex vivo (Kisling et al., 1993; Wakabayashi et al., 1995) studies. Much fewer studies address the acute circulatory fates of cadmium, particularly in term of the cardiovascular regulatory mechanisms in brain.

By passing the blood-brain barrier (BBB) and accumulating in the central nervous system (CNS), cadmium may induce neurotoxicity (Wang and Du, 2013) by acting directly on the central circulatory regulatory mechanisms. One potential target of cadmium is the rostral ventrolateral medulla (RVLM), a key neural substrate in the baroreflex neural circuit that is intimately involved in the maintenance of stable blood pressure and sympathetic vasomotor tone (Dampney, 1994; Spyer, 1994). Chang et al. (2009) demonstrated that bioenergetic failure, leading to necrotic cell death, accounts for the loss of functional integrity in RVLM. Clinical studies (Kuo et al., 1997b; Yien et al., 1997; Yen et al., 2000) demonstrated that the resultant defunct baroreflex-mediated sympathetic vasomotor tone is causally related to brain stem death in comatose patients.

The brain uses $\sim 20 \%$ of total body's oxygen for normal function, making tight regulation of blood flow and oxygen delivery to the brain critical for survival (Widmaier et al., 2006; Bélanger et al., 2011). As such, cerebral autoregulation, which plays an important role in the maintenance of constant blood flow to brain, is another potential target for cadmium-induced neurotoxicity. Of particular interest is that the degree of tissue hypoxia in RVLM is a crucial determinant of the severity of brain stem circulatory regulatory dysfunction (Chang et al., 2009; Chan et al., 2011; Li et al., 2012), which is dependent on whether necrosis or apoptosis has ensued (Chang et al., 2009; Li et al., 2012).

The present study assessed the hypothesis that cadmium mediates dose-dependent acute circulatory fates via differential participation of the cardiovascular regulatory mechanisms in brain. Our physiological and biochemical results showed that a lower-dose of cadmium elicited low mortality, transient decrease in arterial pressure, and hypoxia and apoptosis in RVLM that reflect sustained cerebral autoregulation. On the other hand, a higher-dose of cadmium induced high mortality with a short latency by effecting cardiovascular collapse via anoxia, diminished tissue perfusion, mitochondrial dysfunction and bioenergetics failure that echo failure of cerebral autoregulation, leading to necrosis in RVLM.

\section{MATERIALS AND METHODS}

\section{Experimental Animals}

All experimental procedures carried out in this study were approved by the Institutional Animal Care and Use Committee of the Kaohsiung Chang Gung Memorial Hospital (approval number: 2017091402), and were in accordance with the guidelines for animal care and use set forth by that committee. Adult male Sprague-Dawley rats (255-322 g; $n=159)$ purchased from BioLASCO, Taiwan, were used. They were housed in an AAALAC International-accredited Center for Laboratory Animals, with maintained room temperature $\left(24 \pm 1^{\circ} \mathrm{C}\right)$ and $12 \mathrm{~h}: 12 \mathrm{~h}$ light/dark cycle (light on at 06:00). Standard laboratory rat chow and tap water were available ad libitum.

\section{Recording of Hemodynamic Parameters}

As in our previous studies (Tsai et al., 2016, 2017b), animals were initially anesthetized with an induction dose of pentobarbital sodium (50 mg/kg), given intraperitoneally, to carry out tracheotomy for intubation of the trachea, and cannulation of a femoral artery or vein. Maintenance of anesthetic level was provided by intravenous infusion of propofol (Fresenius Kabi, Graz, Austria) at $25 \mathrm{mg} / \mathrm{kg} / \mathrm{h}$. Arterial pressure (AP) recorded from the femoral artery was subjected to continuous, on-line and real-time spectral analysis (Notocord, Croissy-sur-Seine, France). We were particularly interested in the low-frequency (BLF; 0.25$0.8 \mathrm{~Hz}$ ) band, which takes origin from RVLM (Kuo et al., 1997a), and its power density reflects the prevalence of baroreflexmediated sympathetic vasomotor tone (Li et al., 2001; Poon et al., 2016). Heart rate (HR) was derived from the AP signals. During the recording session, animals were allowed to breathe spontaneously with room air. The head of the animal was fixed to a stereotaxic head holder (Kopf, Tujunga, CA, United States), and the body temperature was maintained at $37^{\circ} \mathrm{C}$ by a heating pad.

\section{Intravenous Administration of Cadmium}

Cadmium solutions were prepared by dissolving cadmium acetate (Sigma-Aldrich, St. Louis, MO, United States) in 0.9\% saline. The $\mathrm{pH}$ of cadmium solution was 7.4. To avoid drug accumulation, each animal received a single dose of cadmium (4 or $8 \mathrm{mg} / \mathrm{kg}$ ) via the femoral vein. These doses were selected from preliminary experiments as representative doses that elicited 
differential effects on our experimental parameters within $10 \mathrm{~min}$ after administration.

\section{Measurement of Blood Flow}

Carotid blood flow (CBF) was recorded by a transit-time blood flowmeter (Transonic, Ithaca, NY, United States). The transonic flow probe (1.0 $\mathrm{mm} \mathrm{V-series)} \mathrm{was} \mathrm{placed} \mathrm{around} \mathrm{a} \mathrm{carotid} \mathrm{artery}$ for blood flow recording. The volume flow and the real-time pulsatile flow were recorded.

\section{Measurement of Tissue Oxygen Level, Microvascular Perfusion, and Temperature}

A combined oxygen/temperature/blood flow probe designed for simultaneous and continuous measurement of tissue oxygen tension, blood flow and temperature (Oxford Optronix, Abingdon, United Kingdom) was stereotaxically positioned into RVLM (Chang et al., 2009). Instantaneous changes in local oxygen tension, compensated for fluctuations in tissue temperature, were processed by an OxyLite monitor (Oxford Optronix). Real-time microvascular red blood cell perfusion in tissue was processed by an OxyFlo monitor (Oxford Optronix). The laser Doppler signals from the tissue were recorded in blood perfusion units (BPU), which is a relative unit defined against a controlled motility standard.

\section{Collection of Tissue Samples}

At the conclusion of the recording sessions, animals were perfused with warm saline for the collection of medullary tissues (Tsai et al., 2016, 2017b). A slice of medulla oblongata that contains RVLM (0.5-1.5 mm rostral to the obex) was obtained, and tissues from both sides of the ventrolateral medulla were subsequently collected by micropunches made with a $1 \mathrm{~mm}$ (i.d.) stainless steel bore to cover the anatomical boundaries of RVLM, and were stored immediately in liquid nitrogen.

\section{Determination of Apoptotic Cell Death and Activated Caspase-3}

Rostral ventrolateral medulla tissue samples were homogenized for the determination of apoptotic cell death using a cell death ELISA kit (Roche, Mannheim, Germany) that measured the level of histone-associated DNA fragments in cytoplasm. A caspase- 3 colorimetric assay kit (BioVision, Mountain View, CA, United States) was used to quantify activated caspase-3.

\section{Measurement of ATP Content and ADP/ATP Ratio}

Total ATP level from homogenized RVLM tissue samples was measured by an ATP detection assay kit (Cayman, Ann Arbor, MI, United States) according to the manufacturer's instructions. Light emitted from a luciferase-mediated reaction and measured by a luminometer (Berthold Centro LB 960, Bad Wildbad, Germany) was used to calculate the measured values.

$\mathrm{ADP} / \mathrm{ATP}$ ratio was measured by an $\mathrm{ADP} / \mathrm{ATP}$ ratio assay kit (Abcam, Cambridge, MA, United States) based on bioluminescent detection of ADP and ATP levels; ADP level was measured by its conversion to ATP. According to the manufacturer's recommendation, we collected fresh RVLM tissue samples and prepared single cell suspensions within $30 \mathrm{~min}$ before the assay, and all procedure was conducted at $4^{\circ} \mathrm{C}$. The $\mathrm{ADP} / \mathrm{ATP}$ ratio was determined by [Data D-Data C]/[Data BData A]. Data A, background signal of reaction mix; Data B, sample signal $\sim 2$ min after addition of cells to reaction mix (for the ATP generated by the cells when they are incubated with mix); Data C, sample signal prior to addition of ADP converting enzyme to cells (for the total ATP released and present in the sample); Data $D$, sample signal $\sim 2$ min after addition of ADP converting enzyme to cells (after conversion of ADP from the samples to ATP).

\section{Histological Examination}

As in our previous studies (Su et al., 2016; Tsai et al., 2017a), the brain stem was fixed in $4 \%$ paraformaldehyde for $24 \mathrm{~h}$. The tissues were dehydrated, embedded in paraffin, sectioned at a thickness of $4 \mu \mathrm{m}$, and stained by hematoxylin and eosin for histopathological examination. The stained brain stem sections were digitally scanned with a slide Scanner (Pannoramic MIDI, 3DHistech, Budapest, Hungary).

\section{In situ Detection of Apoptosis}

For in situ detection of apoptosis in the brain stem, a Click-iT Plus TUNEL assay with Alex Fluor 488 dyes (Life Technologies, Carlsbad, CA, United States) was used to process free-floating frozen sections of medulla oblongata for double immunofluorescence staining. According to the manufacturer's instructions, TUNEL assay dye and a mouse monoclonal antiserum (Life Technologies) against a specific neuronal marker, neuron-specific nuclear protein $(\mathrm{NeuN})$, were first added, followed by incubation with a goat anti-mouse I gG conjugated with Alexa Fluor 568 (Life Technologies). Images were viewed with an Olympus Fluoview 1000 (Tokyo, Japan) laser scanning confocal microscope.

\section{Flow Cytometry}

As described previously (Mattiasson et al., 2003; Zhang L. et al., 2016) with modifications, fresh RVLM tissue was washed with ice-cold phosphate-buffered saline and homogenized with Kontes Dounce homogenizer. The homogenate was placed into microcentrifuge tubes to isolate mitochondria by following the instructions of a Mitochondria isolation kit for tissue (Pierce, Rockford, IL, United States). $20 \mu \mathrm{g}$ of isolated mitochondria were stained with nonyl acridine orange (NAO; $100 \mathrm{nM}, \mathrm{Ex} / \mathrm{Em}$ : $488 \mathrm{~nm} / 525 \mathrm{~nm}$; Life Technologies) to evaluate mitochondrial mass and $1,1^{\prime}, 3,3,3^{\prime}, 3^{\prime}$-hexamethylindodicarbocyanine iodide [DiIC 1 (5); 10 nM, Ex/Em: 638 nm/658 nm, Life Technologies] to verify mitochondrial membrane potential. Stained mitochondria were detected by Gallios flow cytometer (Beckman Coulter, Indianapolis, IN, United States), and data were analyzed by Kaluza software (Beckman Coulter). To exclude debris, samples were gated based on light-scattering properties in the forward-scattered light (FS) and side-scattered light 
(SS) modes, and 20,000 events for each sample within the gate were collected.

\section{Measurement of NF- $\kappa B$ Activation}

Activation of the transcription factor NF- $\mathrm{kB}$ was measured by a sensitive colorimetric assay (TransAM NF- $\mathrm{KB}$ p65; Active Motif; Carlsbad, CA, United States) according to the manufacturer's protocol. Briefly, nuclear protein extracted from RVLM was incubated with an immobilized oligonucleotide containing the NF- $\kappa B$ consensus-binding site. This was followed by incubation with a primary NF- $\mathrm{B}$ p 65 antibody, and a secondary peroxidaseconjugated antibody at room temperature. After a colorimetric reaction, the optical density was read at $450 \mathrm{~nm}$ using an ELISA microtiter plate reader.

\section{Western Bolt Analysis}

The concentration of proteins extracted from RVLM was determined by the BCA assay (Pierce). Western blot analysis was carried out on IL-8 (Abcam, Cambridge, MA, United States) and $\beta$-actin (Chemicon, Temecula, CA, United States). Specific antibody-antigen complex was detected using an enhanced chemiluminescence western blot detection system. The amount of protein level was expressed as the ratio relative to $\beta$-actin protein.

\section{Blood Analysis}

Blood samples were collected after cadmium administration. $0.1 \mathrm{ml}$ of arterial blood was used to determine $\mathrm{pH}$, sodium and potassium by the epoc Blood Analysis System (Siemens, Erlangen, Germany). $0.1 \mathrm{ml}$ of plasma was subjected to detection of thrombomodulin level using an ELISA assay (BlueGene Biotech; Shanghai, China).

\section{Statistical Analysis}

All values are expressed as mean \pm SEM. One-way or twoway analysis of variance with repeated measures, as appropriate, were used to assess group means, followed by Fisher Exact test, Tukey test or Scheffé multiple range test for post hoc assessment of individual means. $P<0.05$ were considered statistically significant.

\section{RESULTS}

\section{Cadmium Acutely and Dose-Dependently Reduces Survival}

Intravenous administration of cadmium elicited a dosedependent decrease in survival rate (Figure 1). At the lower-dose $(4 \mathrm{mg} / \mathrm{kg}$, iv), cadmium induced a progressive reduction in survival rate to $89.6 \%$ during the first $10 \mathrm{~min}$ of observation. Increasing the dose to $8 \mathrm{mg} / \mathrm{kg}$ (iv) further diminished the survival rate to $14.3 \%$ by $10 \mathrm{~min}$ post-cadmium. Survival rate was not affected by intravenous administration of saline (vehicle control).

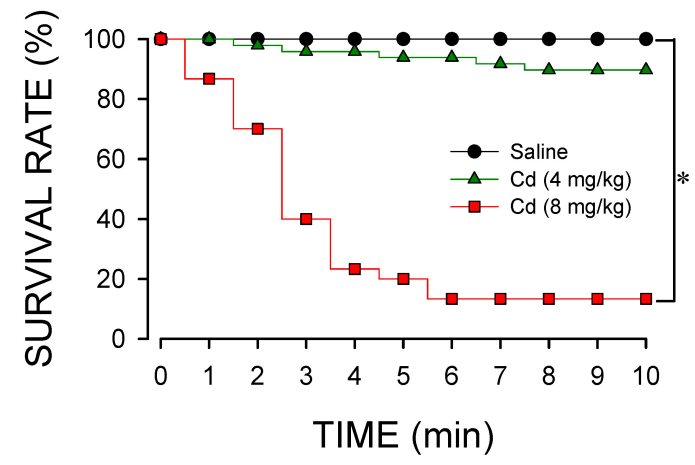

FIGURE 1 | Temporal changes in survival rate in animals that received intravenous administration of cadmium (Cd; 4 or $8 \mathrm{mg} / \mathrm{kg}$ ) or saline.

$n=30-48$ animals per experimental group. ${ }^{*} P<0.05 \mathrm{vs}$. saline group in the Fisher Exact test.

\section{Cadmium Induces Differential and Dose-Related Changes in Arterial Pressure, Heart Rate, or Carotid Blood Flow}

Data shown in Figure 2 demonstrated that intravenous administration of cadmium (4 or $8 \mathrm{mg} / \mathrm{kg}$ ) elicited differential and dose-related changes in AP, HR, or CBF. Administration of the lower-dose of cadmium ( $4 \mathrm{mg} / \mathrm{kg}$, iv) elicited a transient hypotension, accompanied by insignificant changes in HR. Concurrently, cadmium significantly augmented the power density of the BLF component of the AP spectrum to reflect the response of the baroreflex-mediated sympathetic vasomotor tone, along with significantly increased CBF. On the other hand, administration of the higher-dose of cadmium $(8 \mathrm{mg} / \mathrm{kg}$, iv) resulted in a progressive reduction in mean AP (MAP), HR, and CBF (Figure 2) that paralleled temporally with the decline in survival rate (Figure 1). The BLF power underwent a transient, albeit insignificant increase, followed by a steady decline toward zero level that also paralleled temporally with survival rate. The cardiovascular parameters, however, were not affected by intravenous administration of saline (vehicle control).

\section{Higher-Dose of Cadmium Induces Anoxia and Cessation of Tissue Perfusion in RVLM}

Previous study (Chang et al., 2009) demonstrated that anoxia and cessation of tissue perfusion in RVLM precede sustained cessation of BLF power and baroreflex dysfunction seen during experimental brain stem death. Intriguingly, our third series of experiments (Figure 3) showed that animals that succumbed to the higher-dose of cadmium ( $8 \mathrm{mg} / \mathrm{kg}$, iv) also manifested an abrupt anoxia and cessation of local microvascular perfusion in RVLM, followed by a drop in tissue temperature. We also noted that the decrease in local blood flow and tissue oxygen level in RVLM (Figure 3) took place before the decline in BLF power (Figure 2). On the other hand, administration of the lower-dose of cadmium ( $4 \mathrm{mg} / \mathrm{kg}$, iv) induced only a transient 


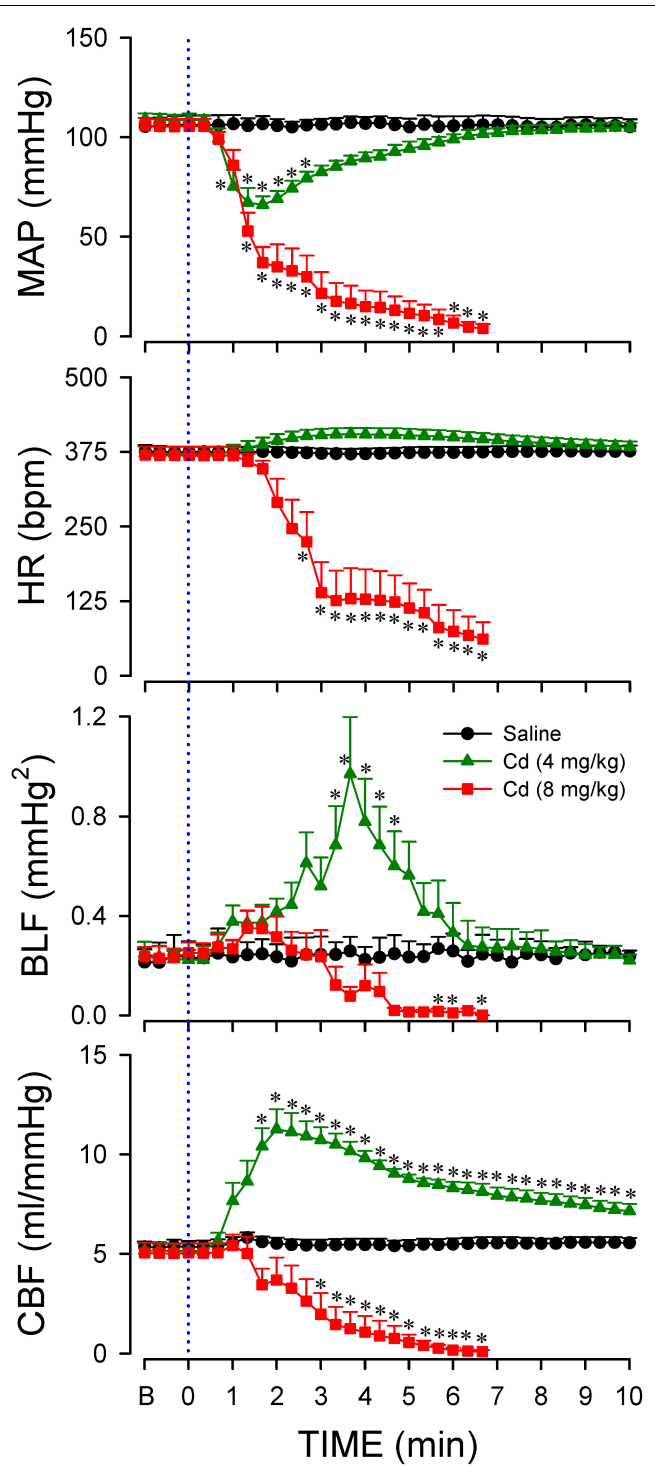

FIGURE 2 | Temporal changes in mean arterial pressure (MAP), heart rate $(\mathrm{HR})$, power density of the low-frequency (BLF) component of arterial pressure spectrum, or carotid blood flow (CBF) in animals that received intravenous administration of cadmium (Cd; 4 or $8 \mathrm{mg} / \mathrm{kg}$ ) or saline. Values are mean \pm SEM, $n=6-8$ animals per experimental group. ${ }^{*} P<0.05$ vs. saline group at corresponding time points in the post hoc Scheffé multiple-range test. Blue dotted line denotes time of cadmium injection. B denotes baseline values.

hypoxia that paralleled the MAP response, alongside insignificant changes in local microvascular perfusion or tissue temperature. Administration of saline induced insignificant changes in tissue oxygen level, local blood flow or tissue temperature in RVLM.

\section{Higher-Dose of Cadmium Induces Necrosis and Lower-Dose of Cadmium Induces Apoptosis in RVLM}

Our hematoxylin and eosin staining results revealed that animals that received the higher-dose of cadmium (Figures 4G,I)

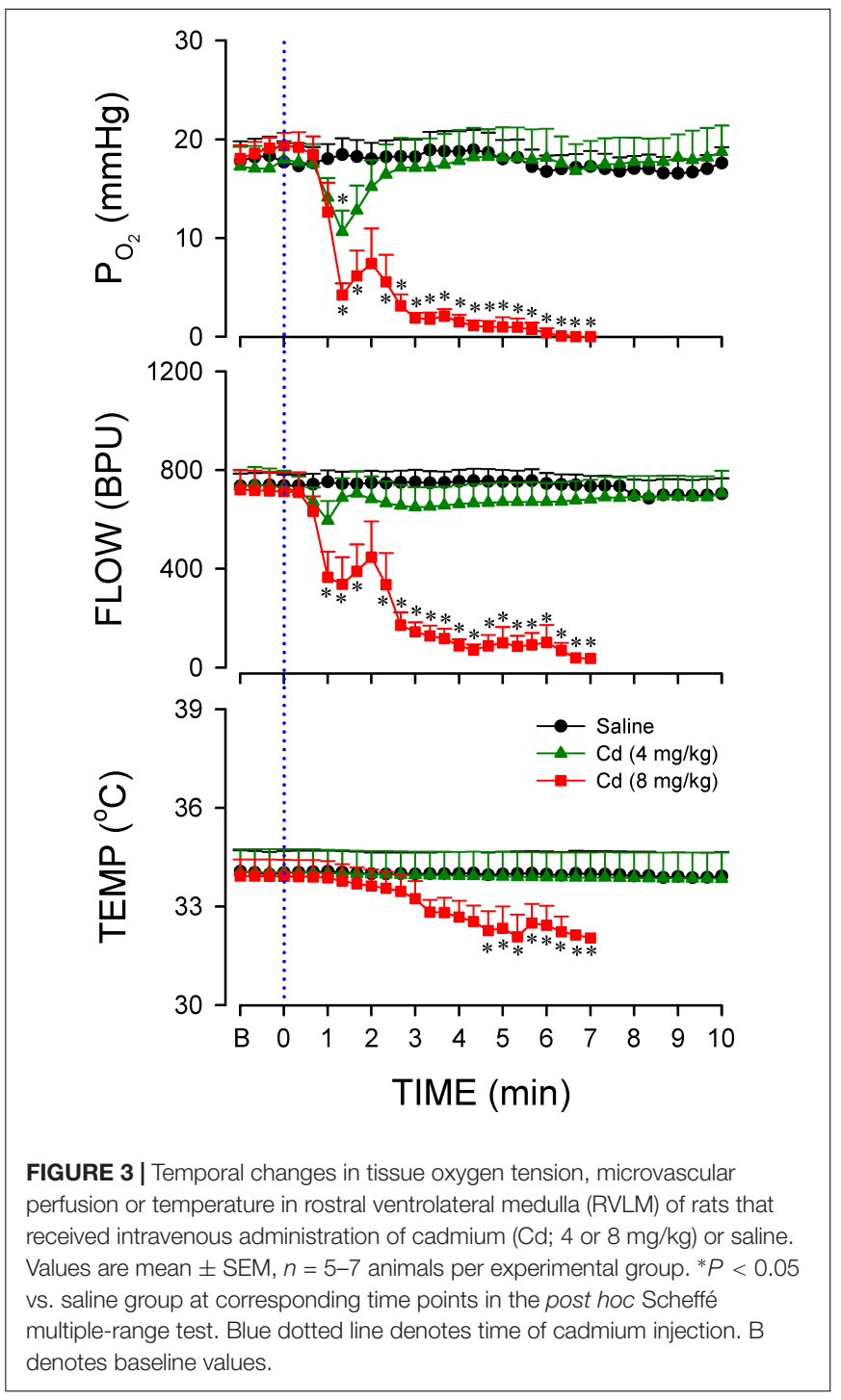

showed necrotic appearing neurons in RVLM, characterized by eosinophilicity (shrunken neurons with loss of normal cytoplasmic and nuclear details, and darkly stained red eosinophilic cytoplasm), karyolysis (nuclear fading), and karyorrhexis (nuclear fragmentation). Quantitative analysis (Figure 4J) revealed that $80.0 \pm 2.6 \%$ of neurons in RVLM of saline-control rats exhibited clear hematoxylin-labeled nucleolus, intact nuclear membrane, nucleus and cytoplasm (Figures $4 \mathrm{~A}-\mathrm{C}$ ), as opposed to $76.7 \pm 5.8 \%$ in the lower-dose cadmium group (Figures 4D-F), and $61.6 \pm 8.6 \%(P<0.05$ vs. saline) in the higher-dose cadmium group (Figures $4 \mathrm{G}-\mathbf{I}$ ). In addition, in animals that received the higher dose of cadmium ( $8 \mathrm{mg} / \mathrm{kg}$, iv), the number of RVLM neurons that manifested eosinophilicity $(17.8 \pm 4.7 \%$ vs. $9.6 \pm 2.5 \% ; P<0.05)$ and karyolysis $(18.9 \pm 3.6 \%$ vs. $7.6 \pm 2.2 \% ; P<0.05)$ was significantly higher than that in the saline-control group. In contrast, results from activated caspase- 3 and histone-associated DNA fragments, two experimental indices for apoptosis, showed that the level of apoptotic cell death in RVLM from the lower-dose cadmium 

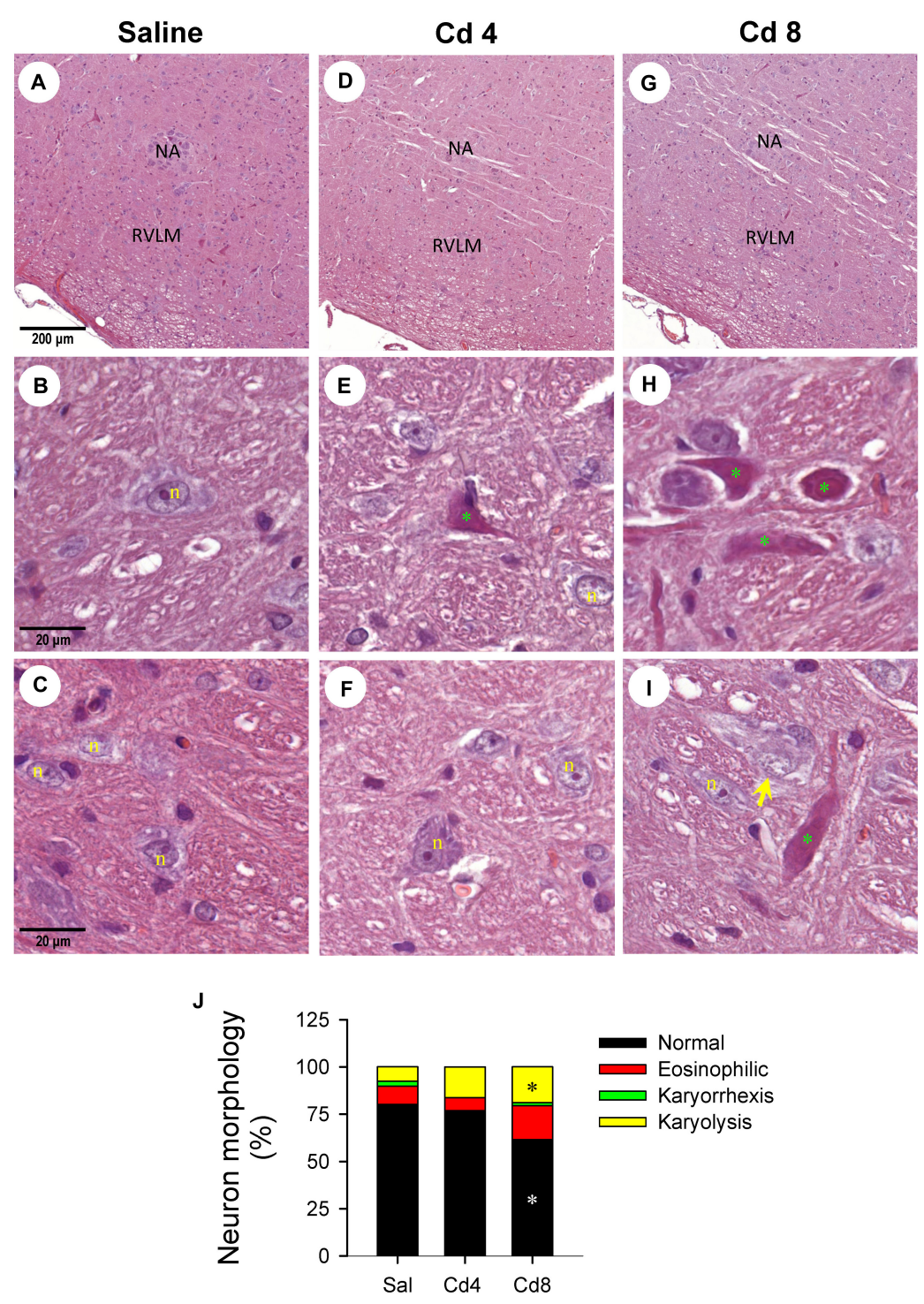

FIGURE 4 | Representative photomicrographs of RVLM stained by hematoxylin and eosin showing nuclear karyolysis and eosinophilic neurons in rats that received saline (A-C), $10 \mathrm{~min}$ after cadmium (Cd; $4 \mathrm{mg} / \mathrm{kg}$, iv) (D-F), or died from Cd (8 mg/kg, iv) (G-I). (J) Stacked bar plots showing the average distribution of neurons in RVLM (in percentage) based on morphology from hematoxylin and eosin staining. These results are typical of $3-4$ animals from each experimental group. ${ }^{*} P<0.05$ vs. saline (sal) group. In (A-I), NA, nucleus ambiguus; n, nucleus; green symbol (*), eosinophilic neuron; yellow arrow, neuron exhibiting karyolysis.

group was significantly augmented (Figure 5). Our results from TUNEL assay (Figure 6) further showed that the increase of apoptotic cell death after administration of cadmium took place in RVLM neurons. However, apoptosis was absent in RVLM of rats that received saline or the higher-dose cadmium.

\section{Bioenergetic Failure Underpins Higher-Dose of Cadmium-Induced Necrotic Cell Death in RVLM}

Since ATP is the primary source of metabolic energy in brain, neurons tend to undergo necrosis in response to hypoxic or anoxic stress (Nagańska and Matyja, 2001). In this series of experiments, we found that rats that received the higher-dose of cadmium ( $8 \mathrm{mg} / \mathrm{kg}$, iv) exhibited a drastic reduction in total ATP level and an increase in ADP/ATP ratio in RVLM (Figure 7). ADP level in RVLM also increased in the high-dose of cadmium group (data not show). However, there was only a slight reduction in total ATP level and no significant alteration in ADP/ATP ratio in RVLM in the lower-dose of cadmium (4 mg/kg, iv) group.

\section{Cadmium Induces Decrease of Mitochondrial Membrane Potential in RVLM}

We further evaluated mitochondrial function in RVLM by measuring changes in mitochondrial membrane potential using flow cytometry. Double staining of mitochondria with NAO 

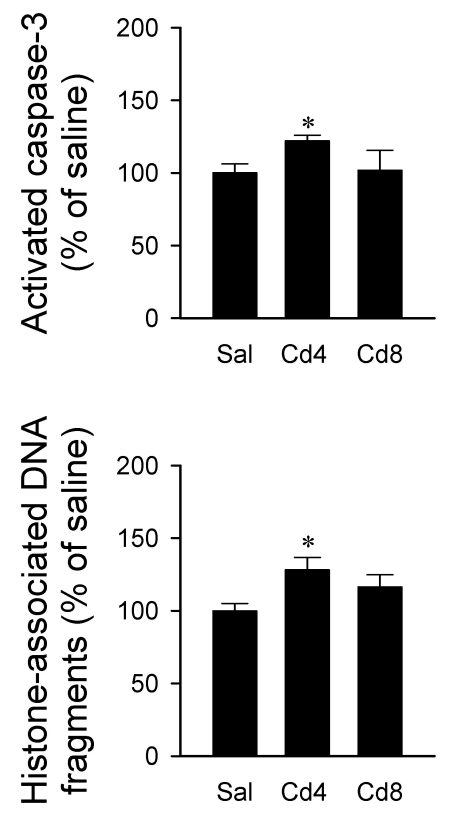

FIGURE 5 | Changes in activated caspase-3 level or histone-associated DNA fragments in cytoplasm against saline-control in tissues collected from RVLM of rats that received saline, 10 min after cadmium (Cd; $4 \mathrm{mg} / \mathrm{kg}$, iv), or died from Cd (8 mg/kg, iv). Values are mean \pm SEM, $n=4-5$ animals per experimental group. ${ }^{*} P<0.05$ vs. saline (sal) group in the post hoc Tukey test.

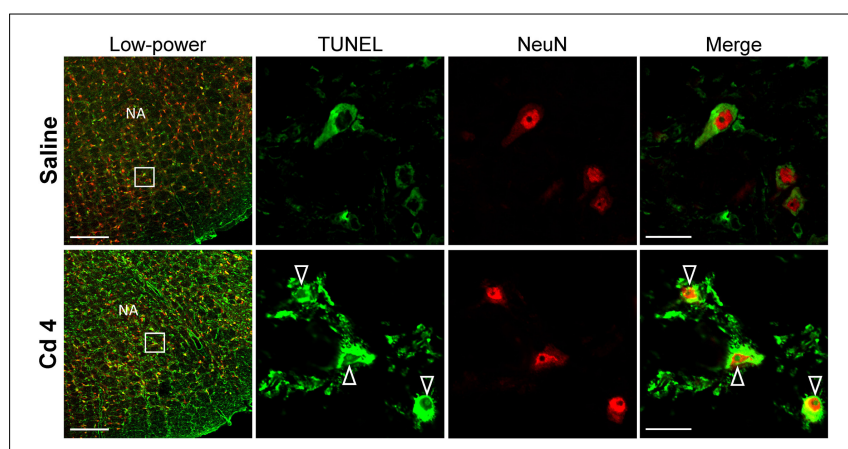

FIGURE 6 | Illustrative examples of in situ detection of apoptosis in RVLM neurons using TUNEL assay (green fluorescence) and staining for the neuronal marker, NeuN (red fluorescence) in rats 10 min after saline or cadmium (Cd; $4 \mathrm{mg} / \mathrm{kg}$, iv). White arrows denote TUNEL-positive staining in the nucleus of cells that additionally exhibit NeuN-immunoreactivity. Box in low-power view of the medulla oblongata indicated the location of TUNEL-positive neurons. Scale bar, $20 \mu \mathrm{m}$ in low-power view or $200 \mu \mathrm{m}$ in high-power view. NA, nucleus ambiguus.

(mitochondrial marker) and $\operatorname{DiIC}_{1}(5)$ (mitochondrial membrane potential indicator) was performed to measure membrane potential only in NAO-stained particles (Figure 8B). In all samples, mitochondria were selected from background based on light-scattering properties in the SS and FS modes (R1; Figure 8A). We found that mitochondrial membrane potential measured by the mean $\operatorname{DiIC}_{1}(5)$ fluorescence intensity (Figure 8C) was significantly reduced in the higher-dose of cadmium groups. However, there was only a slight and no
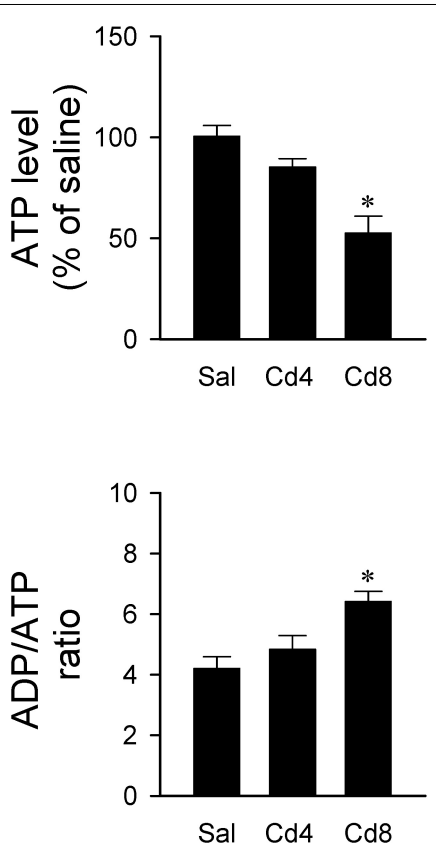

FIGURE 7 | Changes in ATP level against saline-control or ADP/ATP ratio in tissues collected from RVLM of rats that received saline, 10 min after cadmium (Cd; $4 \mathrm{mg} / \mathrm{kg}$, iv), or died from Cd (8 mg/kg, iv). Values are mean \pm SEM, $n=4-5$ animals per experimental group. ${ }^{*} P<0.05$ vs. saline (sal) group in the post hoc Tukey test.

significant reduction in mitochondrial membrane potential in the lower-dose of cadmium group.

\section{Lower-Dose of Cadmium Induces Neuroinflammation in RVLM}

Results from transcriptional activity and western blot analysis (Figure 9) showed that there was no significant alteration in NF$\kappa \mathrm{B}$ activation and IL-8 protein level in RVLM 10 min after loweror higher-dose of cadmium administration. However, animals that received the lower-dose of cadmium showed a significant increase in NF- $\kappa \mathrm{B}$ activation and IL-8 protein expression in RVLM $2 \mathrm{~h}$ after cadmium administration.

\section{Lack of Changes in Blood pH, Sodium and Potassium, or Plasma Level of Thrombomodulin After Acute Administration of Cadmium}

Analysis of blood samples (Table 1) collected $5 \mathrm{~min}$ after the administration of the lower- or higher-dose of cadmium showed no significant changes in blood $\mathrm{pH}$, sodium and potassium or plasma level of thrombomodulin.

\section{DISCUSSION}

A majority of the reported mechanisms for cadmium-induced cardiovascular toxicity is based on either long-term exposure to cadmium in animals (Puri, 1999; Yoopan et al., 2008; 
A

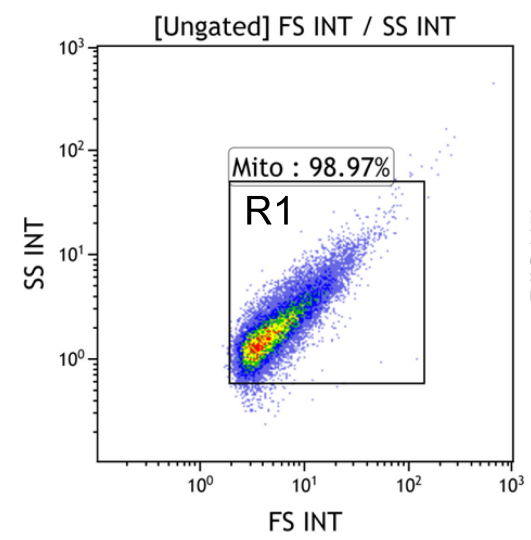

FS INT

B

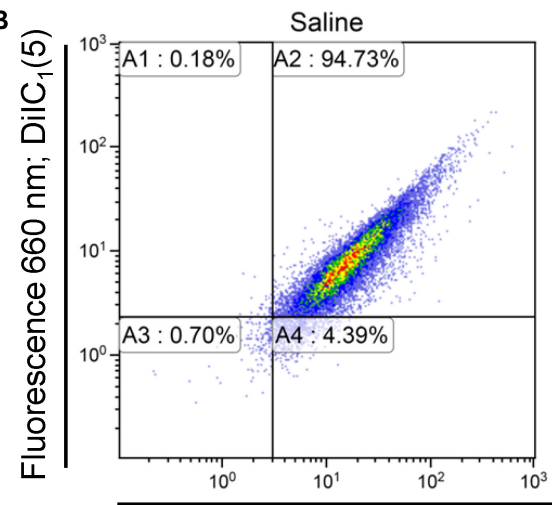

C

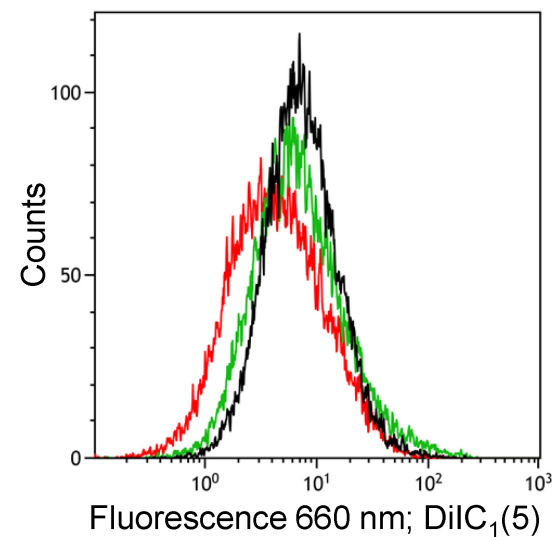

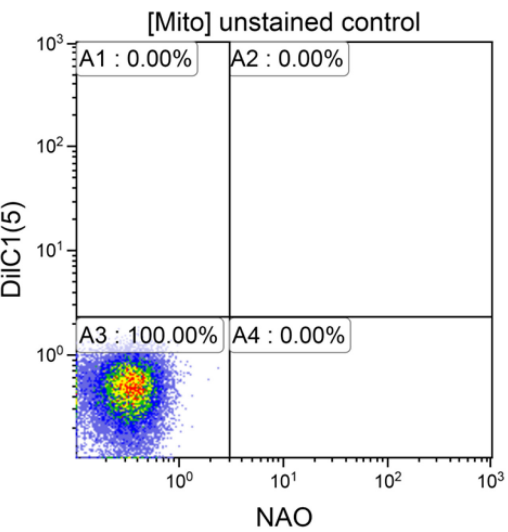
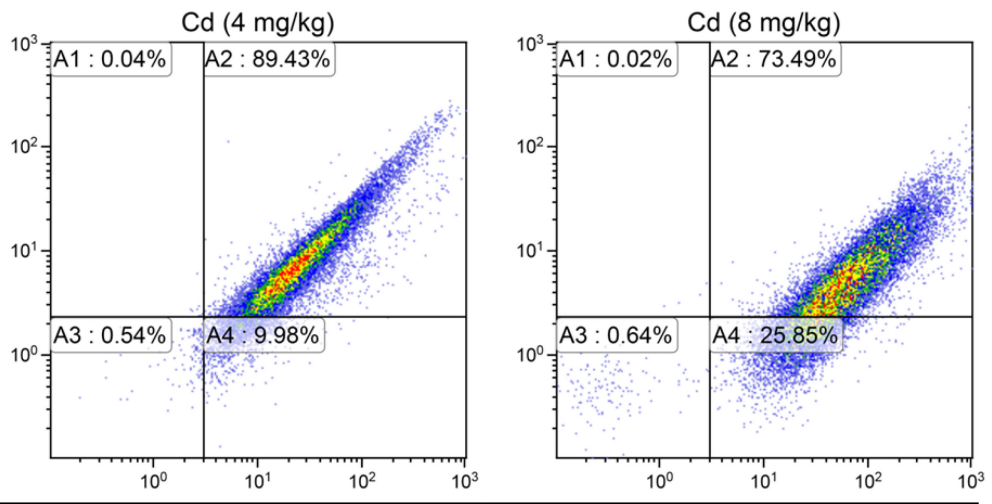

Fluorescence $525 \mathrm{~nm}$; NAO
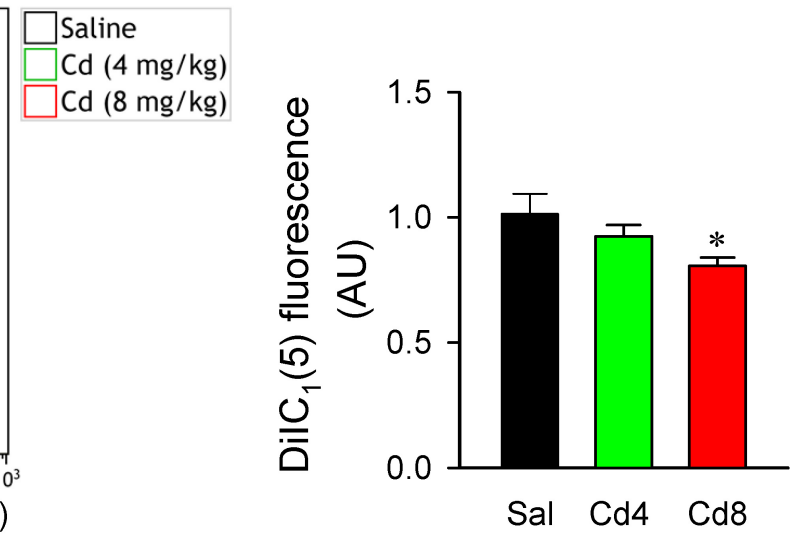

FIGURE 8 | Estimation of mitochondrial membrane potential from freshly isolated mitochondria of RVLM tissue. (A) Mitochondria were gated based on light scattering properties in the FS and SS modes by 20,000 events for each sample within the gate R1. Unstained control sample shown fluorescence at 525 and $660 \mathrm{~nm}$ for the events within R1. (B) Representative dot plots from a double-staining using $100 \mathrm{nM} \mathrm{NAO}$ (mitochondrial marker) and $10 \mathrm{nM}$ DilC ${ }_{1}(5)$ (mitochondrial membrane potential indicator). (C) Representative histogram or quantification of mean intensity of fluorescence at DilC ${ }_{1}(5)(660 \mathrm{~nm})$ from mitochondria in rats that received saline, $10 \mathrm{~min}$ after cadmium (Cd; $4 \mathrm{mg} / \mathrm{kg}$, iv), or died from Cd (8 mg/kg, iv). Values are mean $\pm \mathrm{SEM}, n=5-6$ animals per experimental group. ${ }^{*} P<0.05$ vs. saline (sal) group in the post hoc Tukey test.

Ozturk et al., 2009), or from ex vivo (Kisling et al., 1993; Wakabayashi et al., 1995) or in vitro (Majumder et al., 2008; Messner and Bernhard, 2010; Messner et al., 2016) studies. Much less studies address the acute circulatory actions of cadmium. The present study fills this void by providing physiological and biochemical observations which revealed that acute exposure to cadmium resulted in drastically different dose-dependent circulatory fates that entail differential engagement of central cardiovascular regulatory mechanisms. 

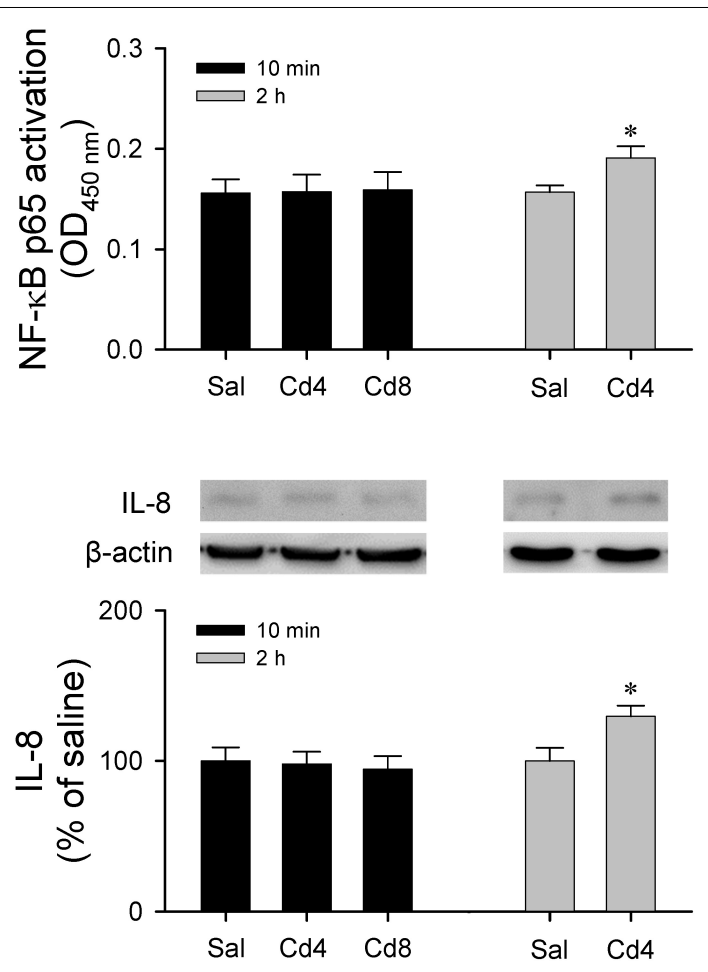

FIGURE 9 | Measurement of transcriptional activity of NF-кB p65 in the RVLM or western blot analysis of expression of $\mathrm{IL}-8$ relative to $\beta$-actin in tissues collected from RVLM of rats that received saline, 10 min after cadmium (Cd; $4 \mathrm{mg} / \mathrm{kg}$, iv), or died from Cd (8 mg/ $\mathrm{kg}$, iv). Values are mean $\pm \mathrm{SEM}, n=3-4$ animals per experimental group. ${ }^{*} P<0.05$ vs. saline (sal) group in the post hoc Tukey test.

Previous studies (Kuo et al., 1997b; Chan et al., 2005) demonstrated that disappearance of the baroreflex-medicated sympathetic vasomotor tone precedes brain stem death in comatose patients who succumbed to severe brain injury (Yien et al., 1997), systemic inflammatory response syndrome (Kuo et al., 1997b), or organophosphate poisoning (Yen et al., 2000). The present results showed that a time-window represented experimentally by the power density of the BLF component also exists between the administration of cadmium and death, which was inversely related to the doses used. On administration of the higher-dose of cadmium, our results showed that blood pressure dropped quickly in the first min after injection, BLF sustained for $3 \mathrm{~min}$, and the animal finally died after $10 \mathrm{~min}$. However, under a lower-dose of cadmium, the MAP, HR, and BLF were maintained to reflect high survival rate.

The brain uses $20 \%$ of available oxygen for normal function, making tight regulation of blood flow and oxygen delivery critical for survival (Widmaier et al., 2006; Bélanger et al., 2011). Flow autoregulation is the mechanism by which organs and tissues alter their own arteriolar resistances, thereby selfregulating their blood flow (Widmaier et al., 2006). Our results from $\mathrm{CBF}$ measurements also showed dose-dependent changes in cerebral autoregulation that paralleled survival after cadmium administration. Intriguingly, the increased in CBF after administration of the lower-dose cadmium was reflected in sustained tissue oxygen level and local blood flow in RVLM, which peaked before maximal BLF response occurred. On the other hand, the progressive decrease in CBF was echoed by a gradual decline in tissue oxygen, local blood flow, tissue temperature and BLF power, leading to high mortality rate after administration of the higherdose of cadmium.

The brain is highly dependent on energy for normal activity, and mitochondria are the major source of ATP production. By revealing similar patterns of reduction in ATP level and mitochondrial membrane potential, our results suggest that cadmium induces mitochondrial dysfunction that leads to decrease of ATP production in RVLM. Previous studies (Yen et al., 2004; Chang et al., 2009) demonstrated that mitochondrial dysfunction in RVLM is a major culprit of baroreflex dysregulation. It follows that mitochondrial dysfunction in brain stem sites involved in cardiovascular regulation remains to be a potential target for cadmium toxicity.

In vitro study showed that whereas lower concentrations of cadmium $(<1 \mu \mathrm{M})$ induce apoptosis, higher concentrations of cadmium $(>1 \mu \mathrm{M})$ induce necrotic cell death (López et al., 2003). Furthermore, intracellular ATP levels have been implicated as a determinant for apoptosis or necrosis (Tsujimoto, 1997; Tatsumi et al., 2003). It is therefore of interest that our histological staining results revealed that the higher-dose of cadmium induced necrotic cell death in RVLM neurons, along with significant decrease in ATP level. In contrast, with only slight reduction of ATP level, rats that received the lower-dose of cadmium exhibited an increase in activated caspase- 3 and histone-associated DNA fragments in RVLM, and results from TUNEL assay indicated the occurrence of apoptotic cell death in RVLM neurons. However, we recognize that both neurons and astrocytes can release ATP during basal metabolic activity and during neuronal activation (Shetty et al., 2012). It is therefore possible for apoptotic cell death to take place in neurons and astrocytes in RVLM.

Necrotic cell death occurs in response to many kinds of insults (e.g., trauma, infarction, and toxins, etc.) and therefore is typically the result of a pathological process (Rock and Kono, 2008). Morphologically, it is associated with cell swelling and/or the rapid loss of membrane integrity and an uncontrolled release of products of cell death into the

TABLE 1 | Analyses of blood samples collected from rats 5 min after intravenous administration of cadmium (Cd; 4 or $8 \mathrm{mg} / \mathrm{kg}$ ) or saline.

\begin{tabular}{lccc}
\hline & Saline & Cd $\mathbf{4} \mathbf{~ m g} / \mathbf{k g})$ & $\mathbf{C d}(\mathbf{8} \mathbf{~ m g} / \mathbf{k g})$ \\
\hline $\mathrm{pH}$ & $7.37 \pm 0.03$ & $7.35 \pm 0.01$ & $7.40 \pm 0.01$ \\
Sodium $(\mathrm{mmol} / \mathrm{L})$ & $142.3 \pm 0.3$ & $140.3 \pm 0.8$ & $142.3 \pm 0.8$ \\
Potassium $(\mathrm{mmol} / \mathrm{L})$ & $4.00 \pm 0.05$ & $4.23 \pm 0.28$ & $4.10 \pm 0.20$ \\
Thrombomodulin $(\mathrm{pg} / \mathrm{mL})$ & $182 \pm 16$ & $193 \pm 10$ & $185 \pm 22$
\end{tabular}

Values are mean $\pm S E M, n=3$ animals per experimental group. No significance among all groups $(P>0.05)$ in ANOVA analysis. 
extracellular space (Proskuryakov et al., 2003). In contrast to apoptosis, necrosis is almost always detrimental and can be fatal. Chang et al. (2009) demonstrated that bioenergetic failure leading to necrotic cell death accounts for the loss of functional integrity in RVLM, leading to failure to resume spontaneous circulation after cardiac arrest in experimental brain stem death. They also used tissue oxygen level as a determinant of functional integrity of RVLM. Our results showed that the drop of local blood flow or tissue oxygen level in RVLM occurred earlier than the cardiovascular responses and before animals succumbed to the higherdose of cadmium. When combined with our ATP and histological staining results, these observations support that bioenergetic failure, leading to necrotic cell death, accounts for the loss of functional integrity in RVLM, and death in animal. In the present study, acute intoxication was induced by intravenous administration of cadmium. It is therefore possible that cadmium may also affects the afferent component of baroreflex such as baroreceptors located in aortic arch and carotid sinus or the nucleus tractus solitarii where baroreceptor afferents terminate in the brain stem (Dampney, 1994; Spyer, 1994).

Cadmium is a potent inhibitor of voltage-dependent calcium channel (Yang et al., 1993). Previous study (Yuan et al., 2013) reported that cadmium elicits neurotoxicity through disruption of intracellular free calcium homeostasis, leading to apoptosis via calcium-mitochondria signaling. Our flow cytometry results also showed a reduction of mitochondrial membrane potential, suggesting mitochondrial dysfunction in RVLM. It is therefore conceivable that cadmium may induce cardiovascular impairment via calcium-mitochondria signaling in RVLM neurons. We also noted that voltagedependent calcium channels expressed in chemoreceptor cells of rabbit carotid body have been shown to be affected by cadmium (Rocher et al., 2005). Our results from blood sample analysis showed that the blood $\mathrm{pH}$ in animals was not significantly changed at $5 \mathrm{~min}$ after intravenous administration of lower- or higher-dose of cadmium. The contribution of chemoreflex to our observations is therefore deemed minimal.

Cadmium can pass the BBB and accumulate in the CNS via increasing permeability of BBB (Shukla et al., 1996) to induced neurotoxicity (Wang and $\mathrm{Du}, 2013$ ). It has also been reported that cadmium stimulates intercellular adhesion molecule-1 (ICAM-1) (Jeong et al., 2004) expression via NF- $\mathrm{B}$ activation in cerebrovascular endothelial cells, leading to BBB injury. Recent studies showed that cadmium induces neuroinflammation via activation of astrocytes or microglia (Ashok et al., 2015; Favorito et al., 2017). Phuagkhaopong et al. (2017) also reported that the cadmium-induced interleukin 6 (IL-6) and interleukin 8 (IL-8) expression and release from astrocytes are mediated by NF- $\mathrm{KB}$ at 6 and $24 \mathrm{~h}$ post-exposure of cadmium. Our results showed that there was no significant alteration in NF$\kappa \mathrm{B}$ activation and IL-8 protein level in RVLM $10 \mathrm{~min}$ after lower- or higher-dose of cadmium administration. However, animals that received the lower-dose of cadmium showed a significant increase in NF- $\mathrm{KB}$ activation and IL-8 protein expression in RVLM after $2 \mathrm{~h}$ of cadmium administration. The causal relationship between neuroinflammation and BBB injury in RVLM and impairment of cardiovascular regulation during cadmium-induced sub-lethal cardiovascular toxicity remains to be established in the future.

Epidemiological studies from humans (Nakagawa and Nishijo, 1996; Tellez-Plaza et al., 2008) and studies in animals (Puri, 1999; Yoopan et al., 2008) have revealed that chronic exposure to cadmium causes hypertension. Its mechanism has been reported to include endothelial dysfunction (Lukkhananan et al., 2015), suppression of eNOS expression in endothelial cells (Majumder et al., 2008) or in blood vessels (Yoopan et al., 2008), and vascular relaxation (Yoopan et al., 2008). Of note is that our results showed no significant changes in plasma thrombomodulin, a marker for endothelial dysfunction (John et al., 1999), at 5 min after administration of the lower- or higherdose of cadmium. The contribution of endothelial dysfunction to our results on acute cardiovascular effects of cadmium is therefore deemed minimal.

The kidney is another organ of injury after cadmium exposure (Winiarska-Mieczan and Kwiecień, 2016); hyperkalemia associated with renal injury was reported in rats $5 \mathrm{~h}$ after intravenous administration of cadmium (Dote et al., 2007). In addition, previous study in human renal glomerular endothelial cells indicated that NF- $\mathrm{kB}$ signaling plays an essential role in maintaining the survival of low-dose cadmium exposed (Zhang H. et al., 2016). However, our results showed no significant alterations in NF- $\mathrm{kB}$ activation (Figure 9) or blood electrolytes (Table 1) 5-10 min after cadmium administration. As such, it is unlikely that our observed dose-dependent acute cardiovascular effects induced by cadmium are related to renal injury.

We are cognizant that there are at least two limitations in the present study. First, it is difficult to carry out realtime detection of cadmium concentrations in the RVLM. Second, cadmium has been administered in different routes and doses for chronic or acute exposure studies in humans, animals or in vitro preparations. The doses of cadmium used in the present study were adopted from the report by Puri (1999) and selected from preliminary experiments as representative doses that elicited differential effects on our experimental parameters within $10 \mathrm{~min}$ after administration.

In conclusion, the present study provided physiological and biochemical evidence that support the notion that cadmium elicits drastically different circulatory fates that are dose-dependent and entail differential engagement of central cardiovascular regulatory mechanisms. We demonstrated that a lower-dose of cadmium elicited low mortality, transient decrease in MAP, and hypoxia and apoptosis in RVLM neurons that reflect sustained cerebral autoregulation. In contrast, a higher-dose of cadmium induced acute cardiovascular collapse by the loss of functionality in RVLM because of anoxia, diminished tissue perfusion, mitochondrial dysfunction and bioenergetic failure that echo loss of cerebral autoregulation, leading to necrosis that further exacerbated mortality. 


\section{ETHICS STATEMENT}

All experimental procedures carried out in this study were approved by the Institutional Animal Care and Use Committee of the Kaohsiung Chang Gung Memorial Hospital (Approval Number: 2017091402), and were in accordance with the guidelines for animal care and use set forth by that committee.

\section{AUTHOR CONTRIBUTIONS}

S-MC collected, analyzed, and interpreted the data, and drafted the manuscript. SP, CF, JW, and Y-HH collected, analyzed, and interpreted the data. PV and H-HL conceived and designed the study and critically revised the intellectual content of the manuscript. C-YT conceived and designed the study, critically revised the intellectual content of the manuscript, supervised the overall study, and drafted the manuscript. All authors revised and approved the final version of the manuscript.

\section{REFERENCES}

Ashok, A., Rai, N. K., Tripathi, S., and Bandyopadhyay, S. (2015). Exposure to As-, $\mathrm{Cd}-$, and $\mathrm{Pb}$-mixture induces $\mathrm{A} \beta$, amyloidogenic $\mathrm{APP}$ processing and cognitive impairments via oxidative stress-dependent neuroinflammation in young rats. Toxicol. Sci. 143, 64-80. doi: 10.1093/toxsci/kfu208

Bélanger, M., Allaman, I., and Magistretti, P. J. (2011). Brain energy metabolism: focus on astrocyte-neuron metabolic cooperation. Cell Metab. 14, 724-738. doi: 10.1016/j.cmet.2011.08.016

Chan, J. Y., Chang, A. Y., and Chan, S. H. (2005). New insights on brain stem death: from bedside to bench. Prog Neurobiol. 77, 396-425. doi: 10.1016/j.pneurobio. 2005.11.004

Chan, J. Y., Tsai, C. Y., Wu, C. H., Li, F. C., Dai, K. Y., Sun, E. Y., et al. (2011). Sumoylation of hypoxia-inducible factor- $1 \alpha$ ameliorates failure of brain stem cardiovascular regulation in experimental brain death. PLoS One 6:e17375. doi: 10.1371/journal.pone.0017375

Chang, A. Y., Chan, J. Y., Chuang, Y. C., and Chan, S. H. (2009). Brain stem death as the vital determinant for resumption of spontaneous circulation after cardiac arrest in rats. PLoS One 4:e7744. doi: 10.1371/journal.pone.0007744

Dampney, R. A. (1994). Functional organization of central pathways regulating the cardiovascular system. Physiol. Rev. 74, 323-364. doi: 10.1152/physrev.1994.74. 2.323

Dote, E., Dote, T., Shimizu, H., Shimbo, Y., Fujihara, M., and Kono, K. (2007). Acute lethal toxicity, hyperkalemia associated with renal injury and hepatic damage after intravenous administration of cadmium nitrate in rats. J. Occup. Health 49, 17-24. doi: 10.1539/joh.49.17

Favorito, R., Monaco, A., Grimaldi, M. C., and Ferrandino, I. (2017). Effects of cadmium on the glial architecture in lizard brain. Eur. J. Histochem. 61, 2734. doi: $10.4081 /$ ejh.2017.2734

Jeong, E. M., Moon, C. H., Kim, C. S., Lee, S. H., Baik, E. J., Moon, C. K., et al. (2004). Cadmium stimulates the expression of ICAM-1 via NF-(B activation in cerebrovascular endothelial cells. Biochem. Biophys. Res. Commun. 320, 887-892. doi: 10.1016/j.bbrc.2004.05.218

John, S., Drobnik, W., Lackner, K., and Schmieder, R. E. (1999). Soluble thrombomodulin and endothelial dysfunction in early atherosclerosis. Lancet 354, 1647. doi: 10.1016/s0140-6736(05)77135-x

Kisling, G. M., Kopp, S. J., Paulson, D. J., and Tow, J. P. (1993). Cadmium-induced attenuation of coronary blood flow in the perfused rat heart. Toxicol. Appl. Pharmacol. 118, 58-64. doi: 10.1006/taap.1993.1009

Kuo, T. B., Yang, C. C., and Chan, S. H. (1997a). Selective activation of vasomotor component of SAP spectrum by nucleus reticularis ventrolateralis in rats. Am. J. Physiol. 272, H485-H492.

\section{FUNDING}

This work was supported by research grants from the Chang Gung Medical Foundation (CMRPG8G1401 and CMRPG8G1402 to C-YT).

\section{ACKNOWLEDGMENTS}

SP received a fellowship support from the Development and Promotion of Science and Technology Talents Project under the Royal Government of Thailand Scholarship. We thank Prof. Samuel H. H. Chan for providing critical revision of the manuscript, Ms. Kuang-Yu Dai for her technical assistance in preparing the histological specimens, and Ms. Chiung-Ju Wu for her technical assistance in flow cytometric analysis. We also thank the Core Laboratory for Phenomics and Diagnostics, Department of Medical Research, Kaohsiung Chang Gung Memorial Hospital for providing the slide Scanner (Pannoramic MIDI, 3DHistech) for histological examination.

Kuo, T. B., Yien, H. W., Hseu, S. S., Yang, C. C., Lin, Y. Y., Lee, L. C., et al. (1997b). Diminished vasomotor component of systemic arterial pressure signals and baroreflex in brain death. Am. J. Physiol. Heart Circ. Physiol. 273, H1291H1298.

Li, F. C. H., Yen, J. C., Chan, S. H. H., and Chang, A. Y. W. (2012). Defunct brain stem cardiovascular regulation underlies cardiovascular collapse associated with methamphetamine intoxication. J. Biomed. Sci. 19, 16. doi: 10.1186/14230127-19-16

Li, P. L., Chao, Y. M., Chan, S. H., and Chan, J. Y. (2001). Potentiation of baroreceptor reflex response by heat shock protein 70 in nucleus tractus solitarii confers cardiovascular protection during heatstroke. Circulation 103, 2114-2119. doi: 10.1161/01.cir.103.16.2114

López, E., Figueroa, S., Oset-Gasque, M. J., and González, M. P. (2003). Apoptosis and necrosis: two distinct events induced by cadmium in cortical neurons in culture. Br. J. Pharmacol. 138, 901-911. doi: 10.1038/sj.bjp. 0705111

Lukkhananan, P., Thawonrachat, N., Srihirun, S., Swaddiwudhipong, W., Chaturapanich, G., Vivithanaporn, P., et al. (2015). Endothelial dysfunction in subjects with chronic cadmium exposure. J. Toxicol. Sci. 40, 605-613. doi: $10.2131 /$ jts. 40.605

Majumder, S., Muley, A., Kolluru, G. K., Saurabh, S., Tamilarasan, K. P., Chandrasekhar, S., et al. (2008). Cadmium reduces nitric oxide production by impairing phosphorylation of endothelial nitric oxide synthase. Biochem. Cell Biol. 86, 1-10. doi: 10.1139/o07-146

Mattiasson, G., Friberg, H., Hansson, M., Elmér, E., and Wieloch, T. (2003). Flow cytometric analysis of mitochondria from CA1 and CA3 regions of rat hippocampus reveals differences in permeability transition pore activation. J. Neurochem 87, 532-544. doi: 10.1046/j.1471-4159.2003. 02026.

Messner, B., and Bernhard, D. (2010). Cadmium and cardiovascular diseases: cell biology, pathophysiology, and epidemiological relevance. BioMetals 23, 811-822. doi: 10.1007/s10534-010-9314-4

Messner, B., Türkcan, A., Ploner, C., Laufer, G., and Bernhard, D. (2016). Cadmium overkill: autophagy, apoptosis and necrosis signalling in endothelial cells exposed to cadmium. Cell Mol. Life Sci. 73, 1699-1713. doi: 10.1007/s00018015-2094-9

Miura, N., Yanagiba, Y., Ohtani, K., Mita, M., Togawa, M., and Hasegawa, T. (2012). Diurnal variation of cadmium-induced mortality in mice. J. Toxicol. Sci. 37, 191-196. doi: 10.2131/jts.37.191

Nagańska, E., and Matyja, E. (2001). Ultrastructural characteristics of necrotic and apoptotic mode of neuronal cell death in a model of anoxia in vitro. Folia Neuropathol. 39, 129-139. 
Nakagawa, H., and Nishijo, M. (1996). Environmental cadmium exposure, hypertension and cardiovascular risk. J. Cardiovasc. Risk. 3, 11-17. doi: 10. 1097/00043798-199602000-00003

Ozturk, I. M., Buyukakilli, B., Balli, E., Cimen, B., Gunes, S., and Erdogan, S. (2009). Determination of acute and chronic effects of cadmium on the cardiovascular system of rats. Toxicol. Mech. Methods 19, 308-317. doi: 10.1080/ 15376510802662751

Phuagkhaopong, S., Ospondpant, D., Kasemsuk, T., Sibmooh, N., Soodvilai, S., Power, C., et al. (2017). Cadmium-induced IL-6 and IL-8 expression and release from astrocytes are mediated by MAPK and NF- $\mathrm{B}$ pathways. Neurotoxicology 60, 82-91. doi: 10.1016/j.neuro.2017.03.001

Poon, Y. Y., Tsai, C. Y., Cheng, C. D., Chang, A. Y., and Chan, S. H. (2016). Endogenous nitric oxide derived from NOS I or II in thoracic spinal cord exerts opposing tonic modulation on sympathetic vasomotor tone via disparate mechanisms in anesthetized rats. Am. J. Physio.l Heart Circ. Physiol. 311, H555-H562.

Proskuryakov, S. Y., Konoplyannikov, A. G., and Gabai, V. L. (2003). Necrosis: a specific form of programmed cell death? Exp. Cell Res. 283, 1-16. doi: 10.1016/ s0014-4827(02)00027-7

Puri, V. N. (1999). Cadmium induced hypertension. Clin. Exp. Hypertens 21, 79-84. doi: 10.3109/10641969909068651

Rocher, A., Geijo-Barrientos, E., Cáceres, A. I., Rigual, R., González, C., and Almaraz, L. (2005). Role of voltage-dependent calcium channels in stimulussecretion coupling in rabbit carotid body chemoreceptor cells. J. Physiol. 562, 407-420. doi: 10.1113/jphysiol.2004.075523

Rock, K. L., and Kono, H. (2008). The inflammatory response to cell death. Annu. Rev. Pathol. 3, 99-126.

Shetty, P. K., Galeffi, F., and Turner, D. A. (2012). Cellular links between neuronal activity and energy homeostasis. Front. Pharmacol. 3:43. doi: 10.3389/fphar. 2012.00043

Shukla, A., Shukla, G. S., and Srimal, R. C. (1996). Cadmium-induced alterations in blood-brain barrier permeability and its possible correlation with decreased microvessel antioxidant potential in rat. Hum. Exp. Toxicol. 15, 400-405. doi: 10.1177/096032719601500507

Spyer, K. M. (1994). Central nervous mechanisms contributing to cardiovascular control. J. Physiol. 47, 1-19. doi: 10.1113/jphysiol.1994.sp019997

Su, C. H., Tsai, C. Y., Chang, A. Y., Chan, J. Y., and Chan, S. H. (2016). MRI/DTI of the brain stem reveals reversible and irreversible disruption of the baroreflex neural circuits: clinical implications. Theranostics 6, 837-848. doi: 10.7150/ thno. 14837

Tatsumi, T., Shiraishi, J., Keira, N., Akashi, K., Mano, A., Yamanaka, S., et al. (2003). Intracellular ATP is required for mitochondrial apoptotic pathways in isolated hypoxic rat cardiac myocytes. Cardiovasc. Res. 59, 428-440. doi: 10.1016/s0008-6363(03)00391-2

Tellez-Plaza, M., Navas-Acien, A., Crainiceanu, C. M., and Guallar, E. (2008). Cadmium exposure and hypertension in the 1999-2004 National Health and Nutrition Examination Survey (NHANES). Environ. Health Perspect. 116, 5156. doi: 10.1289/ehp.10764

Tsai, C. Y., Li, F. C. H., Wu, C. H. Y., Chang, A. Y. W., and Chan, S. H. H. (2016). Sumoylation of IкB attenuates NF-кB-induced nitrosative stress at rostral ventrolateral medulla and cardiovascular depression in experimental brain death. J. Biomed. Sci. 23, 65.

Tsai, C. Y., Su, C. H., Chan, J. Y. H., and Chan, S. H. H. (2017a). Nitrosative stress-induced disruption of baroreflex neural circuits in a rat model of hepatic encephalopathy: a DTI study. Sci. Rep. 7, 40111. doi: 10.1038/srep40111

Tsai, C. Y., Wu, J. C. C., Fang, C., and Chang, A. Y. W. (2017b). PTEN, a negative regulator of PI3K/Akt signaling, sustains brain stem cardiovascular regulation during mevinphos intoxication. Neuropharmacology 123, 175-185. doi: 10.1016/j.neuropharm.2017.06.007

Tsujimoto, Y. (1997). Apoptosis and necrosis: intracellular ATP level as a determinant for cell death modes. Cell Death Differ. 4, 429-434. doi: 10.1038/ sj.cdd.4400262

Wakabayashi, I., Sakamoto, K., and Hatake, K. (1995). Inhibitory effects of cadmium ion on extracellular $\mathrm{Ca} 2+$-independent contraction of rat aorta. Eur. J. Pharmacol. 293, 133-140. doi: 10.1016/s0014-2999(05)80005-6

Wang, B., and Du, Y. (2013). Cadmium and its neurotoxic effects. Oxid. Med. Cell. Longev. 2013, 898034. doi: 10.1155/2013/898034

Widmaier, E. P., Raff, H., and Strang, K. T. (2006). Vander's Human Physiology. The Mechanisms of Body Function, 10th Edn. New York, NY: McGraw-Hill, 204-205,418-423.

Winiarska-Mieczan, A., and Kwiecień, M. (2016). The effect of exposure to Cd and $\mathrm{Pb}$ in the form of a drinking water or feed on the accumulation and distribution of these metals in the organs of growing Wistar rats. Biol. Trace Elem. Res. 169, 230-236. doi: 10.1007/s12011-015-0414-4

Yang, J., Ellinor, P. T., Sather, W. A., Zhang, J. F., and Tsien, R. W. (1993). Molecular determinants of $\mathrm{Ca} 2+$ selectivity and ion permeation in L-type $\mathrm{Ca} 2+$ channels. Nature 366, 158-161. doi: 10.1038/366158a0

Yen, D. H., Chan, J. Y., Tseng, H. P., Huang, C. I., Lee, C. H., Chan, S. H., et al. (2004). Depression of mitochondrial respiratory enzyme activity in rostral ventrolateral medulla during acute mevinphos intoxication in the rat. Shock 21, 358-363. doi: 10.1097/00024382-200404000-00011

Yen, D. H., Yien, H. W., Wang, L. M., Lee, C. H., and Chan, S. H. (2000). Spectral analysis of systemic arterial pressure and heart rate signals of patients with acute respiratory failure induced by severe organophosphate poisoning. Crit. Care Med. 28, 2805-2811. doi: 10.1097/00003246-200008000-00021

Yien, H. W., Hseu, S. S., Lee, L. C., Kuo, T. B., Lee, T. Y., and Chan, S. H. (1997). Spectral analysis of systemic arterial pressure and heart rate signals as a prognostic tool for the prediction of patient outcome in intensive care unit. Crit. Care Med. 25, 258-266. doi: 10.1097/00003246-199702000-00011

Yoopan, N., Watcharasit, P., Wongsawatkul, O., Piyachaturawat, P., and Satayavivad, J. (2008). Attenuation of eNOS expression in cadmium-induced hypertensive rats. Toxicol. Lett. 176, 157-161. doi: 10.1016/j.toxlet.2007.11.002

Yuan, Y., Jiang, C. Y., Xu, H., Sun, Y., Hu, F. F., Bian, J. C., et al. (2013). Cadmiuminduced apoptosis in primary rat cerebral cortical neurons culture is mediated by a calcium signaling pathway. PLoS One 8:e64330. doi: 10.1371/journal.pone. 0064330

Zhang, H., Li, L., Wang, Y., Dong, F., Chen, X., Liu, F., et al. (2016). NF-кB signaling maintains the survival of cadmium-exposed human renal glomerular endothelial cells. Int. J. Mol. Med. 38, 417-422. doi: 10.3892/ijmm.2016.2640

Zhang, L., Trushin, S., Christensen, T. A., Bachmeier, B. V., Gateno, B., Schroeder, A., et al. (2016). Altered brain energetics induces mitochondrial fission arrest in Alzheimer's disease. Sci. Rep. 6, 18725. doi: 10.1038/srep 18725

Conflict of Interest Statement: The authors declare that the research was conducted in the absence of any commercial or financial relationships that could be construed as a potential conflict of interest.

Copyright (C) 2019 Chen, Phuagkhaopong, Fang, Wu, Huang, Vivithanaporn, Lin and Tsai. This is an open-access article distributed under the terms of the Creative Commons Attribution License (CC BY). The use, distribution or reproduction in other forums is permitted, provided the original author(s) and the copyright owner(s) are credited and that the original publication in this journal is cited, in accordance with accepted academic practice. No use, distribution or reproduction is permitted which does not comply with these terms. 